PROCEEDINGS OF THE

AMERICAN MATHEMATICAL SOCIETY

Volume 134, Number 12, December 2006, Pages 3445-3447

S 0002-9939(06)08440-1

Article electronically published on June 9, 2006

\title{
A SIMPLE PROOF OF ZAGIER DUALITY FOR HILBERT MODULAR FORMS
}

\author{
D. CHOI
}

(Communicated by Ken Ono)

\begin{abstract}
In this paper, we give a simple proof of an identity between the Fourier coefficients of the weakly holomorphic modular forms of weight 0 arising from Borcherds products of Hilbert modular forms and those of the weakly holomorphic modular forms of weight 2 satisfying a certain property.
\end{abstract}

\section{INTRODUCTION AND RESULTS}

A certain sequence of modular forms of weight $1 / 2$ arises in the theory of Borcherds products for modular forms with Heegner divisors. Zagier proved that there exists a duality, called Zagier duality, between the Fourier coefficients of these modular forms and those of some modular forms of weight 3/2. In [2] Rouse obtained an analog of Zagier duality between the Fourier coefficients of the weakly holomorphic modular forms of weight 0 arising from Borcherds products of Hilbert modular forms and those of the weakly holomorphic modular forms of weight 2 satisfying a certain property. In this paper, we give a simple proof of the identity proved in 2], by constructing a linear relation among the Fourier coefficients of weakly holomorphic modular forms.

Let $\chi_{p}$ be the Dirichlet character $(\dot{p})$. For an integer $k \geq 0$ and $\epsilon= \pm 1$ let $A_{k}^{\epsilon}\left(\Gamma_{0}(p), \chi_{p}\right)$ denote the subspace of weakly holomorphic modular forms $F(z)$ of weight $k$ and character $\chi_{p}$ such that if $F(z)=\sum_{n \in \mathbb{Z}} c(n) q^{n}$, then $c(n)=0$ for $\chi_{p}(n)=-\epsilon$.

Proposition 1.1. Suppose that $f=\sum_{n \in \mathbb{Z}} a_{f}(n) q^{n} \in A_{k(f)}^{\epsilon}\left(\Gamma_{0}(p), \chi_{p}\right)$ and $g=$ $\sum_{n \in \mathbb{Z}} a_{g}(n) q^{n} \in A_{k(g)}^{\epsilon}\left(\Gamma_{0}(p), \chi_{p}\right)$. For $t \in\{0, \infty\}$ let

$$
\left.f\right|_{2} \gamma_{t}=\sum_{n \in \mathbb{Z}} a_{f}^{t}(n) q^{n} \text { and }\left.g\right|_{0} \gamma_{t}=\sum_{n \in \mathbb{Z}} a_{g}^{t}(n) q^{n}
$$

where $\gamma_{0}=\left(\begin{array}{cc}0 & -1 \\ p & 0\end{array}\right)$ and $\gamma_{\infty}=\left(\begin{array}{ll}1 & 0 \\ 0 & 1\end{array}\right)$. If $k(f)+k(g)=2$, then

$$
\sum_{i+j=0}\left(a_{f}^{0}(i) a_{g}^{0}(j)+a_{f}^{\infty}(i) a_{g}^{\infty}(j)\right)=0 .
$$

Received by the editors June 8, 2005 and, in revised form, July 3, 2005.

2000 Mathematics Subject Classification. Primary 11F11; Secondary 11F41.

Key words and phrases. Modular forms, Hilbert modular forms.

This work was partially supported by KOSEF R01-2003-00011596-0.

(C)2006 American Mathematical Society 
Let $p$ be a prime and

$$
s(m)=\left\{\begin{array}{lll}
2 & \text { if } m \equiv 0 \quad(\bmod p) \\
1 & \text { if } m \neq 0 & (\bmod p) .
\end{array}\right.
$$

From Proposition 1.1 we obtain the following theorem that gives a connection between a weakly holomorphic modular form of weight 0 arising in Borcherds products for Hilbert modular forms (see Theorem 6 in [1]) and a certain weakly holomorphic modular form of weight 2 .

Theorem 1.2. Suppose that, for a nonnegative integer $m$ and a positive integer $d$ with $\chi(m) \neq-\epsilon$ and $\chi(d) \neq-\epsilon$, there is a function $F_{d, p}^{\epsilon}(z) \in A_{0}^{\epsilon}\left(\Gamma_{0}(p), \chi_{p}\right)$ such that

$$
F_{d, p}^{\epsilon}(z)=\frac{1}{s(d)} q^{-d}+O(1)=\sum_{n \in \mathbb{Z}} A_{d, p}(n) q^{n}
$$

and $G_{m, p}^{\epsilon}(z) \in A_{2}^{\epsilon}\left(\Gamma_{0}(p), \chi_{p}\right)$ such that

$$
G_{m, p}^{\epsilon}(z)=\frac{1}{s(m)} q^{-m}+O(q)=\sum_{n \in \mathbb{Z}} B_{m, p}(n) q^{n} .
$$

Then

$$
A_{d, p}(m)=-B_{m, p}(d) .
$$

Remark 1.3. Let $S_{k}^{+}\left(\Gamma_{0}(p), \chi_{p}\right)$ denote the subspace of holomorphic cusp forms in $A_{k}^{+}\left(\Gamma_{0}(p), \chi_{p}\right)$. When $\epsilon=1$ and $p \in\{5,13,17\}$, Theorem 1.2 was proved in [2] by the arithmetic of operators for modular forms.

Remark 1.4. If $p=5,13$ or 17 , then for each $m \geq 1$ with $\chi_{p}(m) \neq-1$, there exists a unique $F_{m, p}^{+}(z) \in A_{0}^{+}\left(\Gamma_{0}(p), \chi_{p}\right)$ and a unique $G_{m, p}^{+}(z) \in A_{2}^{+}\left(\Gamma_{0}(p), \chi_{p}\right)$.

Proof of Proposition 1.1. Suppose $G$ is a meromorphic modular form of weight 2 on $\Gamma_{0}(p)$ for a prime $p$. We denote the set of distinct cusps of $\Gamma_{0}(p)$ as $S_{p}=\{\infty, 0\}$. For $\tau \in \mathbb{H} \cup S_{p}$, let $D_{\tau}$ be the image of $\tau$ under the canonical map from $\mathbb{H} \cup S_{p}$ to $X_{0}(p)$, where $\mathbb{H}$ denotes the complex upper half-plane. The residue of $G$ at $D_{\tau}$ on $X_{0}(p)$, denoted by $\operatorname{Res}_{D_{\tau}} G d z$, is well defined since we have a canonical correspondence between meromorphic modular forms of weight 2 on $\Gamma_{0}(p)$ and meromorphic 1forms of $X_{0}(p)$. If $\operatorname{Res}_{\tau} G$ denotes the residue of $G$ at $\tau$ on $\mathbb{H}$, then we obtain

$$
\operatorname{Res}_{D_{\tau}} G d z=\frac{1}{l_{\tau}} \operatorname{Res}_{\tau} G .
$$

Here, $\lambda_{\tau}$ is the order of the isotropy group at $\tau$. The residue of $G$ at each cusp $t$ in $S_{p}$ is

$$
\operatorname{Res}_{D_{t}} G d z=\frac{a_{t}(0)}{2 \pi i},
$$

where $\left.G(z)\right|_{2} \gamma_{t}=\sum_{n=m_{t}}^{\infty} a_{t}(n) q^{n}$ at $\infty$.

To prove Proposition 1.1 we take $G=f g$. Since $\chi_{p}$ is a quadratic character, $G$ is a meromorphic modular form of weight 2 on $\Gamma_{0}(p)$. We have

$$
\left.f g\right|_{2} \gamma_{t}=\left(\sum_{n \in \mathbb{Z}} a_{f}^{t}(n) q^{n}\right)\left(\sum_{n \in \mathbb{Z}} a_{g}^{t}(n) q^{n}\right) .
$$

Therefore, by the residue theorem we give a proof of Proposition 1.1. 
Proof of Theorem 1.2. We begin by stating the following lemma.

Lemma 1.5 (Lemma 3 of [1]). Let $F(z)=\sum_{n \in \mathbb{Z}} A(n) q^{n} \in A_{k}\left(\Gamma_{0}(p), \chi_{p}\right)$ and $\epsilon \in\{1,-1\}$. Then $F(z) \in A_{k}^{\epsilon}\left(\Gamma_{0}(p), \chi_{p}\right)$ if and only if

$$
p^{1-k / 2}\left(F \mid U_{p}\right)=\epsilon \sqrt{p}\left(\left.F\right|_{k} \gamma_{0}\right),
$$

where $F \mid U_{p}=\sum_{n \in \mathbb{Z}} A(p n) q^{n}$.

From Lemma 1.5 we have

$$
\epsilon \cdot\left(F_{d, p}^{\epsilon} \mid 0 \gamma_{0}\right)=p^{\frac{1}{2}} F_{d, p}^{\epsilon} \mid U_{p}=p^{\frac{1}{2}} \frac{s(d)-1}{s(d)} q^{-d / p}+p^{\frac{1}{2}} \sum_{n \geq 0} A_{d, p}(p n) q^{n}
$$

and

$$
\epsilon \cdot\left(\left.G_{m, p}^{\epsilon}\right|_{2} \gamma_{0}\right)=p^{-\frac{1}{2}} G_{m, p}^{\epsilon} \mid U_{p}=p^{-\frac{1}{2}} \frac{s(m)-1}{s(m)} q^{-m / p}+p^{-\frac{1}{2}} \sum_{n \geq 1} B_{m, p}(p n) q^{n} .
$$

Using Proposition 1.1, we have

$$
\left(\frac{1}{s(m)}+\frac{s(m)-1}{s(m)}\right) A_{d, p}(m)+\left(\frac{1}{s(d)}+\frac{s(d)-1}{s(d)}\right) B_{m, p}(d)=0
$$

This proves Theorem 1.2.

Remark 1.6. Following the method used in the proof of Proposition 1.1, one can also obtain the similar identity between the Fourier coefficients of $f_{k_{1}}$ in $A_{k_{1}}^{\epsilon}\left(\Gamma_{0}(p), \chi_{p}\right)$ and those of $f_{k_{2}}$ in $A_{k_{2}}^{\epsilon}\left(\Gamma_{0}(p), \chi_{p}\right)$ where $k_{1}+k_{2}=2$.

\section{ACKNOWLEDGEMENT}

We are very grateful to J. Rouse for giving his preprint to us and for his valuable comments. We also thank the referee for a careful reading of the manuscript and for helpful comments.

\section{REFERENCES}

[1] J. H. Bruinier and M. Bundschuh, On Borcherds products associated with lattices of prime discriminant. Rankin memorial issues, Ramanujan J. 7 (2003), no. 1-3, 49-61. MR2035791 (2005a:11057)

[2] J. Rouse, Zagier duality for the exponents of Borcherds products for Hilbert modular forms, to appear in Journal of the London Mathematical Society.

Department of Mathematics, Pohang University of Science and Technology, Pohang, 790-784, Korea

E-mail address: choija@postech.ac.kr Korea

Current address: School of Mathematics, KIAS, 207-43 Cheongnyangni 2-dong, Seoul, 130-722, 